\title{
Tendência do aleitamento materno em município da região centro-sul do estado de São Paulo: 1995-1999-2004
}

\author{
Breastfeeding trends in a municipality in \\ central-southern São Paulo state: 1995-1999-2004
}

Luciana FERREIRA ${ }^{1}$

Cristina Maria Garcia de Lima PARADA²

Maria Antonieta de Barros Leite CARVALHAES²

\section{R E S U M O}

\section{Objetivo}

Avaliar a situação do aleitamento materno em 2004 no município de Botucatu, SP, e identificar sua tendência nos últimos 10 anos.

\section{Métodos}

Trata-se de um estudo epidemiológico, no qual foram comparados os resultados de 3 inquéritos populacionais transversais (1995-1999-2004), metodologicamente semelhantes, sobre a situação do aleitamento materno em crianças menores de 12 meses. Os dados foram obtidos em Campanhas de Multivacinação, a partir de questionário contendo um recordatório da alimentação das crianças no dia anterior à pesquisa. Para identificar a tendência dos diferentes tipos de aleitamento (aleitamento materno exclusivo, aleitamento materno predominante e aleitamento materno), foram comparadas as prevalências, segundo faixas etárias selecionadas (0-1 mês, 0-4 meses, 0-6 meses e 0-12 meses), nos 3 inquéritos. Os resultados foram submetidos a teste estatístico (teste $z$ ) para verificação das diferenças entre proporções.

\section{Resultados}

Para as crianças menores de 4 meses, houve aumento progressivo e de grande magnitude $(19,1 \%$ em 1995 e 36,9\% em 2004) do aleitamento materno exclusivo e diminuição do aleitamento materno predominante - diferenças estatisticamente significantes. O mesmo ocorreu para as crianças menores de 6 meses: 13,0\% em aleitamento materno exclusivo em 1995, 29,6\% em 2004, representando 128,0\% de aumento. Com relação ao aleitamento materno, tanto para as crianças menores de 4 meses, quanto para as menores de 6 meses e de 1 ano, houve pequeno aumento de prevalência, mas as diferenças não foram estatisticamente significantes. A duração mediana do aleitamento materno exclusivo aumentou 14 dias (82,0\%) e do aleitamento materno 85 dias (50,9\%) no período de 10 anos.

\footnotetext{
1 Universidade Estadual Paulista Júlio de Mesquita Filho, Faculdade de Medicina, Programa de Saúde da Família e Comunidade. Botucatu, SP, Brasil.

2 Universidade Estadual Paulista Júlio de Mesquita Filho, Faculdade de Medicina, Departamento de Enfermagem. Campus Universitário Rubião Júnior, s/n. 18618-970, Botucatu, SP, Brasil. Correspondência para/Correspondence to: C.M.G.L. PARADA. E-mail: <cparada@fmb.unesp.br>.
} 


\section{Conclusão}

Com a elevação expressiva da prevalência de crianças menores de 6 meses em aleitamento materno exclusivo e o aumento da mediana da amamentação exclusiva e da amamentação, pode-se afirmar que a evolução do aleitamento no município foi favorável. Entretanto, a situação em 2004 ainda está distante das recomendações atuais sobre alimentação infantil.

Termos de indexação: aleitamento materno; lactante; prevalência.

\section{A B S T R A C T}

\section{Objective}

To evaluate breastfeeding practices in 2004 in the municipality of Botucatu, São Paulo State and to identify trends in the past ten years.

\section{Methods}

This is an epidemiological study comparing the results of three population-based cross-sectional studies (1995-1999-2004), using similar methodologies, assessing the breastfeeding practices among children under age 12 months. Data were obtained during multiple vaccination campaigns using a recall questionnaire to investigate the child's feeding in the previous day. The differences in the proportion of children in each type of feeding (exclusive breastfeeding, predominant breastfeeding, and breastfeeding) in the three surveys, according to selected age groups (0-1 month, 0-4 months, 0-6 months and 0-12 months), were analyzed using a z-test.

\section{Results}

For children under age 4 months, there was a marked and progressive increase $119.1 \%$ in 1995 and 36.9\% in 2004) in exclusive breastfeeding and a reduction in predominant breastfeeding, with statistically significant differences. The same was observed among children under age 6 months, with 15\% increase in exclusive breastfeeding in 1995 and 29.6\% in 2004, representing a total 128.0\% increase. Regarding breastfeeding, there were slight increases in prevalence for children younger than 4 months, 6 months, and 1 year, but significant differences were not found. The median duration of exclusive breastfeeding increased by 14 days (82.0\%) and that of breastfeeding, by 85 days (50.9\%) in the ten-year period.

\section{Conclusion}

With the expressive increase in the prevalence of exclusive breastfeeding among children under six months and the increase in median duration of exclusive breastfeeding and of breastfeeding, we may conclude that breastfeeding in the municipality evolved favorably. However, the situation in 2004 is still far from fulfilling current recommendations on infant feeding.

Indexing terms: breast feeding; prevalence; infant.

\section{N T R O D U Ç Ã O}

É inquestionável o relevante papel que o aleitamento materno desempenha no crescimento e no desenvolvimento infantil, além de ser parte integrante do processo reprodutivo, com importantes implicações para a saúde materna ${ }^{1}$.

Devido às suas propriedades físico-químicas e especificidades em relação às necessidades nutricionais da criança, o leite materno é o único alimento que garante qualidade e quantidade ideal de nutrientes para o lactente ${ }^{2}$.

Vários estudos mostram que a amamentação exclusiva nos primeiros seis meses de vida reduz a mortalidade infantil por enfermidades comuns da infância, como diarréia e pneumonia ${ }^{3}$, e ajuda na recuperação de enfermidades ${ }^{4-7}$.

Estudo realizado em 1987 já evidenciava que crianças que não recebiam leite materno tinham risco 14 vezes maior de morrer por diarréia e 3,6 vezes maior de mortalidade por doenças respiratórias, quando comparadas às que recebiam apenas leite materno sem complementos ${ }^{6}$.

O aleitamento materno também contribui para a saúde da mulher, protegendo-a contra o câncer de mama ${ }^{8}$, câncer de ovário ${ }^{9}$, osteoporose ${ }^{10}$ e esclerose múltipla ${ }^{11}$. Outra vantagem para a 
saúde da mulher é a de ampliar o espaçamento entre as gestações e promover efeito contraceptivo, pela amenorréia induzida pela lactação (LAM), confiável nos primeiros seis meses após o parto, desde que a amamentação seja exclusiva e que a mãe se mantenha amenorréica ${ }^{12}$.

O aleitamento materno exclusivo oferece a vantagem adicional de diminuir os custos das famílias, dos estabelecimentos de saúde e da sociedade em geral, ao eliminar os gastos com leites artificiais, mamadeiras e ao reduzir os episódios de doenças nas crianças $^{13}$.

Há evidências suficientes que embasam a recomendação da Organização Mundial de Saúde (OMS) de amamentação exclusiva por 6 meses e manutenção do aleitamento materno complementado até os 2 anos ou mais. No entanto, ainda é baixo o número de mulheres que a seguem, estando a duração e a prevalência dessa prática muito aquém do recomendado ${ }^{14}$.

Em 1998, estudo envolvendo 84 municípios do estado de São Paulo evidenciou que o aleitamento materno exclusivo nos primeiros 4 meses, raramente, alcançou índices superiores a $30 \%$ e, entre os menores de 12 meses, a amamentação (aleitamento materno sem ou com outros alimentos) ficou em torno de 50\% ${ }^{15}$.

No Brasil, dados do Ministério da Saúde apontam que a prevalência de aleitamento materno exclusivo, em menores de 1 mês, era de $53,1 \%$ e entre crianças de 3 a 4 meses, $21,6 \%$, decrescendo para 9,7\% entre crianças de 5 a 6 meses $^{16}$.

Se, por um lado, a situação atual ainda não é ideal, por outro é inquestionável a melhora da situação brasileira: se, em 1975, uma em cada duas mulheres amamentava apenas até o segundo ou terceiro mês, em 1999, uma em cada duas mulheres amamentava até cerca de 10 meses $^{17}$.

Conhecer como os índices do aleitamento materno vêm se comportando ao longo dos anos pode ser importante do ponto de vista gerencial, pois permite melhorar o direcionamento de programas de promoção, proteção e apoio. Assim, foi proposto este estudo, cujos objetivos foram avaliar a situação do aleitamento materno no município de Botucatu, SP, no ano de 2004, e identificar sua tendência nos últimos 10 anos.

\section{M É T O D O S}

Trata-se de um estudo epidemiológico, no qual foram comparados os resultados de 3 inquéritos populacionais transversais metodologicamente semelhantes sobre a situação do aleitamento materno em crianças menores de 12 meses no município de Botucatu, SP. O primeiro estudo foi realizado em $1995^{18}$, o segundo em $1999^{19}$ e o terceiro em 2004, cujos dados são apresentados neste artigo.

O município de Botucatu localiza-se na Região Centro-Sul do Estado de São Paulo e contava com uma população de 108.306 habitantes no ano de 2000, com distribuição predominantemente urbana ${ }^{20}$. Nos 3 estudos ora comparados, a população consistiu das crianças menores de 12 meses, que foram levadas a uma das etapas da Campanha de Multivacinação. Estimava-se que 1.782 crianças estivessem na faixa etária de 0 a 12 meses em 2004²1, 1.777 em $1999^{19}$ e 1.736 em 1995'18. Foram obtidos dados válidos sobre a alimentação de 1.239 crianças em 2004, 1.273 crianças em 1999 e 1.509 crianças em 1995, representando, respectivamente, $90,6 \%, 79,9 \%$ e $91,8 \%$ do total de crianças menores de 12 meses vacinadas no dia de cada Campanha.

Para descrever a situação do aleitamento, foram obtidas informações referentes à data de nascimento da criança, situação do aleitamento materno (a criança mama no peito - sim ou não?) e, na vigência dessa prática, investigou-se o consumo de água, suco, chá, outros líquidos, outro leite ou papa salgada (sim ou não?).

As informações referiam-se aos dados atuais da alimentação da criança, os quais permitem uma simplificação do instrumento de coleta de dados, uma vez que não são incluídas questões 
retrospectivas sobre a alimentação infantil como, por exemplo, até quando mamou no peito e quando foram introduzidos outros alimentos. Trabalhando com dados atuais, evitam-se erros decorrentes da imprecisão da informação das mães (erros de memória).

Esse tipo de coleta de dados tem sido muito utilizado em pesquisas que envolvem crianças e mães, por garantir alta cobertura, rápida coleta de dados e baixo custo ${ }^{15,18}$.

De posse desses dados, estudou-se a duração mediana da amamentação e a prevalência de crianças nas seguintes categorias de aleitamento materno, conforme padronização da Organização Mundial da Saúde ${ }^{22}$ :

- Aleitamento Materno Exclusivo (AME): crianças que receberam somente leite materno, sem outros líquidos ou outro leite, nas últimas 24 horas;

- Aleitamento Materno Predominante (AMP): crianças que receberam leite materno e água, chá ou suco, nas últimas 24 horas;

- Aleitamento Materno (AM): crianças que receberam leite materno, independentemente da oferta de qualquer complemento, lácteo ou não, nas últimas 24 horas.

Os dados foram coletados por entrevistadores treinados antes das campanhas, que trabalharam de tal forma a não comprometer o fluxo de crianças a serem vacinadas, evitando-se filas. As mães eram encaminhadas a uma sala previamente preparada para coleta de dados. A supervisão, no dia de cada uma das campanhas, foi realizada pelas autoras.

Nos três estudos, foram adotados os mesmos procedimentos. As entrevistas foram codificadas, sendo criados arquivos de dados utilizando recursos que permitiam apenas a entrada de dados previstos na codificação, para evitar erros de digitação. Os questionários com questões em branco foram revisados, parte dos dados digitados foi conferida e a consistência dos dados foi checada pela verificação da distribuição de freqüências de crianças em categorias de variáveis associadas. Eventuais erros foram corrigidos.

As prevalências e as medianas das várias modalidades de amamentação, com os respectivos intervalos de confiança, foram obtidas por meio da análise de probitos, realizada com os programas Statistical Package for Social Sciences (SPSS) 6.0 (1995 e 1999) e 12.0 (2004). Essa técnica de análise de regressão, adotada em ensaios biológicos do tipo dose-resposta de medicamentos, foi adaptada para estudo da situação do aleitamento materno em 1996²3, e vem sendo bastante utilizada desde então. Para estudo da amamentação, considera-se que o desmame é o evento terminal, sendo a idade da criança o fator de exposição.

As diferenças de proporções de crianças nos diferentes tipos de aleitamento, nos 3 inquéritos, segundo faixas etárias selecionadas ( 0 - 1 mês, 0-4meses, 0-6 meses, 0-12 meses), foram submetidas a teste estatístico (teste $z)^{24}$, teste bicaudal, adotando-se como estatisticamente significantes resultados com valor de $p<0,05$.

As faixas etárias adotadas correspondem às recomendadas pela OMS para estudo da situação populacional do aleitamento materno (0-6 e 0-12) e a faixas de interesse local de monitoramento, ressaltando-se que as categorias não são excludentes e, sim, cumulativas ${ }^{22}$.

As pesquisas foram realizadas mediante autorização da Secretaria Municipal de Saúde de Botucatu, e com a aprovação do Comitê de Ética em Pesquisa da Faculdade de Medicina de Botucatu.

\section{RES U L T A D O S}

Merece ser destacado que a distribuição do conjunto das crianças menores de um ano estudadas em cada ano mostrou, de modo geral, um padrão uniforme nas diferentes faixas de idade.

A situação do aleitamento materno exclusivo, aleitamento materno predominante e aleitamento materno, segundo a faixa etária da criança, 
nos diferentes anos, bem como as diferenças percentuais de prevalências entre 1999 e 1995, 2004 e 1999 e entre 2004 e 1995, e os respectivos valores de $p$ para avaliação da significância estatística dessas diferenças, são apresentados na Tabela 1.

A Tabela 1 mostra, para as crianças menores de 1 mês, pequenas diferenças nas prevalências do aleitamento materno nos três anos estudados. A comparação de 1995 e de 1999 com 2004 evidencia discreta melhora, embora tenha havido uma queda nos índices de 1999 em relação a 1995. O aumento percentual de prevalência na década foi de 13,0\%, e entre 2004 - 1999 foi de $33,3 \%$, mas essas diferenças não alcançaram significância estatística.

$O$ aleitamento materno predominante diminuiu progressivamente na década entre as crianças menores de 1 mês, sendo a diferença percentual de prevalência entre 2004 e 1995 igual a - 37,5\% e entre 2004 e 1999, - 23,9\%. Porém, essas diferenças também não foram estatisticamente significantes.

Pode-se observar, para as crianças menores de 4 meses, aumento progressivo das preva- lências do aleitamento materno exclusivo nos anos subseqüentes à primeira avaliação (1995), sendo esse aumento mais expressivo entre 1999 e 2004, e diminuição progressiva do aleitamento predominante. Todas as diferenças de prevalências relacionadas ao AME em menores de 4 meses foram estatisticamente significantes. Com relação ao aleitamento materno (AM) nessa faixa etária, comparando-se 1995 e 2004, houve pequena elevação da prevalência, sem alcançar significância estatística. Mas, entre 1999 e 2004, a elevação foi estatisticamente significante $(p=0,0315)$.

Entre as crianças menores de 6 meses, as prevalências do aleitamento materno exclusivo foram sempre crescentes, enquanto que do aleitamento predominante foram decrescentes, sendo essas diferenças estatisticamente significantes. A proporção de crianças em AME nessa faixa etária mais do que dobrou na década. $O$ aumento na prevalência do AME para esse grupo de crianças foi mais expressivo no período de 1999 a 2004, como pode ser melhor observado na Figura 1.

A evolução do AM em menores de 6 meses, na década estudada, foi positiva, com

Tabela 1. Prevalência dos diferentes tipos de aleitamento materno em crianças de faixas etárias selecionadas nos anos de 1995, 1999 e 2004. Botucatu, SP, 2004

\begin{tabular}{|c|c|c|c|c|c|c|c|c|c|}
\hline \multirow{3}{*}{ Faixa etária } & \multicolumn{3}{|c|}{ Ano } & \multicolumn{6}{|c|}{ Diferença \% de prevalência e valor de $p$} \\
\hline & \multirow{2}{*}{$1995(\%)$} & \multirow{2}{*}{$1999(\%)$} & \multirow{2}{*}{$2004(\%)$} & \multicolumn{2}{|c|}{$1999-1995$} & \multicolumn{2}{|c|}{$2004-1999$} & \multicolumn{2}{|c|}{$2004-1995$} \\
\hline & & & & $\%$ & $p$ & $\%$ & $p$ & $\%$ & $p$ \\
\hline 0-1 meses & $n=88$ & $n=82$ & $n=80$ & & & & & & \\
\hline AME & 43,2 & 36,6 & 48,8 & $-15,3$ & 0,3800 & 33,3 & 0,12 & 13,0 & 0,120 \\
\hline AMP & 34,1 & 28,0 & 21,3 & $-17,9$ & 0,3900 & $-23,9$ & 0,298 & $-37,5$ & 0,298 \\
\hline AM & 92,1 & 92,7 & 95,0 & 0,6 & 0,8800 & 2,5 & 0,528 & 3,1 & 0,542 \\
\hline 0-4meses & $n=506$ & $n=420$ & $n=412$ & & & & & & \\
\hline AME & 19,1 & 22,6 & 36,9 & 18,3 & 0,0450 & 63,3 & $<0,0001$ & 93,2 & $<0,0001$ \\
\hline AMP & 34,2 & 25,7 & 16,5 & $-24,8$ & 0,0006 & $-35,8$ & 0,001 & $-51,7$ & $<0,0001$ \\
\hline AM & 79,8 & 77,9 & 83,7 & $-2,4$ & 0,4650 & $-7,4$ & 0,0315 & 4,9 & 0,128 \\
\hline 0-6 meses & $n=792$ & $n=643$ & $n=611$ & & & & & & \\
\hline AME & 13,0 & 16,5 & 29,6 & 26,9 & 0,0100 & 79,4 & $<0,0001$ & 127,7 & $<0,0001$ \\
\hline AMP & 29,9 & 19,7 & 12,9 & $-34,1$ & $<0,0001$ & $-34,4$ & $<0,001$ & $-56,7$ & $<0,0001$ \\
\hline AM & 70,3 & 71,8 & 79,2 & 2,1 & 0,6670 & 10,3 & 0,0011 & 12,7 & 0,0002 \\
\hline 0-12 meses & $n=1.509$ & $n=1.273$ & $n=1.235$ & & & & & & \\
\hline AM & 53,5 & 53,0 & 61,7 & & & & & & 01 \\
\hline
\end{tabular}

AME: aleitamento materno exclusivo; AMP: aleitamento materno predominante; AM: aleitamento materno. 
elevações estatisticamente significantes nas comparações 2004 com 1999 e 2004, com 1995. Em 2004, cerca de $80 \%$ das crianças menores de seis meses eram amamentadas, quase $30 \%$ de modo exclusivo.

A prevalência do aleitamento materno em menores de 1 ano manteve-se praticamente a mesma na primeira metade do período, quando comparados os anos de 1995 e 1999, e apresentou pequeno aumento de 1999 a 2004. Na década, a diferença percentual de prevalência foi de 15,3\%, $p<0,001$. A Figura 1 destaca a tendência na década da prevalência de AM em menores de 12 meses.

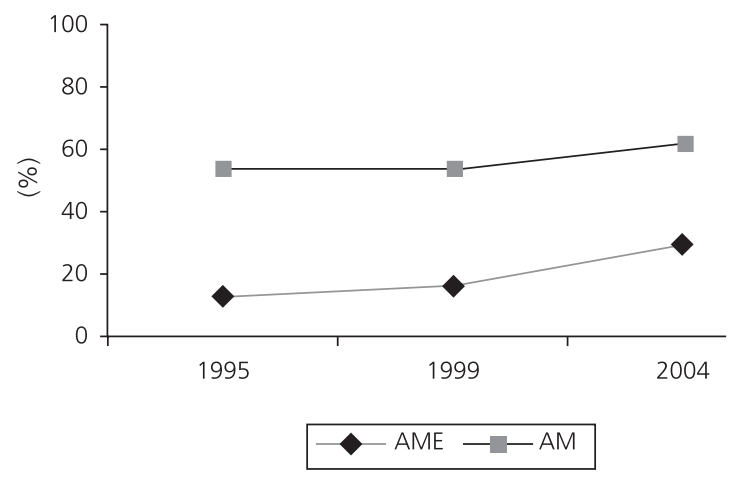

Figura 1. Tendência da prevalência do aleitamento materno exclusivo (AME) em menores de 6 meses e do aleitamento materno (AM) em menores de 12 meses, nos anos de 1995, 1999 e 2004. Botucatu, 2004.

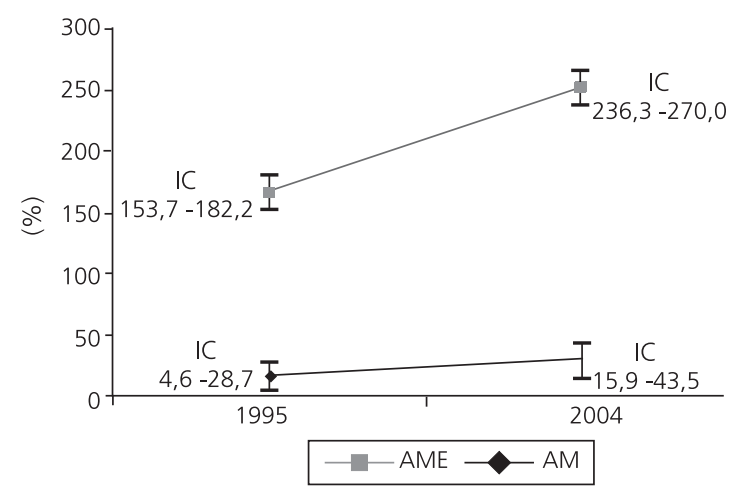

Figura 2. Tendência da duração mediana do aleitamento materno exclusivo e do aleitamento materno (dias), com os respectivos intervalos de confiança (IC), para os anos de 1995 e 2004. Botucatu, SP, 2004.
A duração mediana, com os respectivos intervalos de confiança, do AME e do AM é mostrada na Figura 2. Como essas informações não estão disponíveis para o estudo realizado em 1999, são apresentados, na referida Figura, apenas os dados de 1995 e 2004.

A duração mediana do AME em 1995 foi 17 dias, aumentando para 31 dias em 2004. Assim, a diferença de mediana na década para essa categoria de aleitamento foi 14 dias (aumento percentual de $82,0 \%$ ). Para o aleitamento materno, a mediana aumentou de 167 dias em 1995 para 252 dias em 2004, com diferença de 85 dias (aumento percentual de 50,9\%).

\section{I S C U S S Ã O}

Analisando a tendência das diferentes categorias do aleitamento materno no município de Botucatu, SP, nos últimos 10 anos, pode-se observar que, de uma maneira geral, houve melhora nos indicadores, principalmente para o AME (mais significante), mas também para o AM. A revisão de publicações brasileiras revela que essa mesma tendência foi observada para o País inteiro e em vários municípios nas décadas de 80 e $90^{25}$.

A comparação dos três inquéritos mostrou diferenças pequenas, que não alcançaram significância estatística, nas prevalências de crianças menores de 1 mês em aleitamento materno, seja de modo exclusivo, predominante ou apenas crianças amamentadas (AM). As altas prevalências de AM nesse grupo etário, nos 3 estudos, indicam que praticamente todas as crianças do município iniciam a amamentação após o nascimento. As prevalências de AM nessa faixa etária em Botucatu, SP, nos 3 anos estudados, são um pouco maiores que a encontrada em 2000 (90,3\%) por Montrone \& Arantes $^{26}$, mediante estudo realizado com a mesma metodologia, no município de São Carlos, SP, cidade com porte semelhante ao de Botucatu, SP.

Com relação ao AME em menores de um mês, a situação atual em Botucatu, SP, ainda é 
inferior à de alguns municípios do mesmo estado, como São Carlos, SP26 (52,4\%) e Itapira, SP27 (64,8\%). Em 2004, em Botucatu, SP, mais da metade das crianças nessa idade já recebia água, chás ou outros leites.

Para as crianças com idade até 4 meses, merece destaque o grande aumento na prevalência do AME, já que, em 2004, 36,9\% dessas crianças recebiam exclusivamente leite materno, enquanto que em 1995 a prevalência era de apenas $19,1 \%$. Na verdade, o aumento foi mais expressivo de 1999 para 2004. Estudo realizado em 84 municípios paulistas em 1998, já citado, mostrou que apenas 32,0\% deles apresentavam prevalência de $\mathrm{AME}$, em menores de 4 meses, superior a 20,0\% ${ }^{15}$. Também foi importante a queda na prevalência do AMP, evidenciando uma melhora no nocivo padrão de introdução precoce de outros líquidos à alimentação infantil. A magnitude dessa mudança pode ser percebida quando se avalia a diferença percentual de prevalência do AME nessa faixa etária na década: $93,2 \%$.

Uma possível explicação para a tendência positiva do AME seria a melhoria das práticas de cuidado de mães e recém-nascidos nas maternidades. Esta hipótese se apóia em evidências do estudo sobre situação do aleitamento em municípios paulistas: crianças cujas mães residiam em locais onde inexistia Hospital Amigo da Criança (HAC) tinham 2,2 vezes mais chances de receber precocemente outros líquidos e alimentos nos primeiros quatro meses de vida ${ }^{15}$. Em Botucatu, $\mathrm{SP}$, até o momento, não existe $\mathrm{HAC}$, mas há um movimento nesse sentido, iniciado no final da década de 90 no Hospital das Clínicas, hospital de referência para o parto de médio e alto risco na região e que, pela presença de seus docentes e alunos, também exerce considerável influência em outra maternidade desse município. É possível que esse seja um fator a explicar a evolução positiva do AME em menores de 4 e também em menores de 6 meses, ocorrida principalmente, entre 1999 e 2004. Em relação às crianças menores de 6 meses, merece destaque o aumento expressivo do AME: mais de 127,0\%.
Diante dos dados da literatura ${ }^{6,28}$, pode-se supor que a tendência positiva do AME detectada possa exercer um efeito positivo sobre os coeficientes de morbimortalidade infantil no município.

Para o conjunto de crianças com até 1 ano, a prevalência da amamentação cresceu na segunda metade da década estudada, alcançando, em 2004, 61,7\%. Esse índice é, até certo ponto, surpreendentemente melhor do que o encontrado em Pelotas, RS ${ }^{29}$ no ano de 1993, e em comunidades do Nordeste da Bahia ${ }^{30}$, no período de 1988 a 1989, onde apenas 23,1\% e $12,5 \%$ das crianças nessa faixa etária recebiam leite materno, respectivamente. Entretanto, diferenças na época de realização desses estudos limitam tais comparações.

Uma maneira mais sintética de avaliar a tendência do aleitamento materno é comparar as medianas obtidas na década: houve aumento, passando de 167 dias (5,5 meses) em 1995 para 252 dias (8,4 meses) em 2004, este último valor semelhante ao encontrado em estudos realizados entre 1996 e 1997 em Florianópolis, SC ${ }^{31}$ (238 dias) e Montes Claros, MG ${ }^{32}$ (261 dias) e superior ao encontrado em Ouro Preto, MG no mesmo período (198 dias) ${ }^{33}$.

O último dado brasileiro sobre duração mediana do AM disponível é da Pesquisa Nacional sobre Demografia e Saúde, desenvolvida em São Paulo, Rio de Janeiro e várias regiões do País e que apontou duração mediana do AM de 210 dias $^{25}$. Entretanto, deve-se ponderar que aquela pesquisa foi realizada em 1996 e já apontava tendência de aumento na duração mediana do AM.

Para o aleitamento materno exclusivo, a duração mediana obtida em 2004 foi de 31 dias, 14 dias a mais que a verificada em 1995. Comparando com dados de 1996 e 1997, o valor obtido em 2004, neste estudo, foi superior aos encontrados nas cidades de Montes Claros, MG ${ }^{32}$ (27 dias) e Ouro Preto, MG ${ }^{33}$ (17 dias), mas inferior à mediana de Florianópolis, SC $\left(53\right.$ dias) ${ }^{31}$. Porém, deve ser considerado que, entre 1996 e 2004, a situação nos municípios citados pode ter melhorado, já que essa é a tendência nacional. 
Em síntese, detectou-se expressiva elevação do AME em menores de quatro e de seis meses no período de 1995 a 2004, particularmente entre 1999 e 2004, e elevação mais discreta do AM.

Embora não tenha sido objetivo deste estudo identificar as causas das mudanças ocorridas, podem ter contribuído com a melhoria observada: a criação do Banco de Leite Humano na maternidade terciária do município, em 1999, bem como a intenção desta em se qualificar, com vistas a receber o título de Hospital Amigo da Criança; a mudança na atenção básica do município, com a criação de unidades de Saúde da Família e o aumento no número dos profissionais de saúde envolvidos com a amamentação no município. Tais fatos apóiam evidências da literatura de que, no que diz respeito ao aleitamento materno, o potencial de influência dos serviços e dos profissionais de saúde é grande.

A situação da amamentação encontrada em Botucatu, SP, em 2004, indica padrões melhores do que aqueles verificados para esse município em meados da década de 90, bem como para outros municípios brasileiros nos anos de 1996 e 1997, embora aquém do preconizado pela OMS ${ }^{14}$. leite materno exclusivo até o sexto mês de vida e com alimentos complementares até dois ou mais anos. Assim, apesar da tendência favorável do aleitamento materno em Botucatu, SP, há ainda muito a melhorar, sendo necessário manter as ações de promoção, proteção e incentivo ao aleitamento materno no município.

Estudos de monitoramento das práticas de alimentação infantil devem continuar sendo realizados, pois constituem importante fonte de informação para o planejamento e a avaliação de políticas e programas dirigidos a apoiar e promover o aleitamento materno. Ações específicas para evitar tanto a precoce introdução de líquidos e outros leites nos primeiros meses de vida, quanto para aumentar a duração do aleitamento materno, além do primeiro ano, devem ser implementadas no município.

\section{REFERÊ NCIAS}

1. Organização Pan-Americana de Saúde. Amamentação; 2003 [acesso em: 18 nov 2003]. Disponível em: http://www.opas.org.br

2. Lopes PRA. As vantagens da amamentação. Porque amamentar? In: Rego JD. Aleitamento materno: um guia para pais e familiares. São Paulo: Atheneu; 2002. p.5-21.

3. Vieira GO, Silva TO. Alimentação infantil e morbidade por diarréia. J Pediatr (Rio J). 2003; 79(5): 449-54.

4. Betran AP, Onis M, Lauer JA, Villar J. Ecological study of effect of breastfeeding on infant mortality in Latin America. Br Med J. 2001; 323(7308): 303-6.

5. Hop LT, Gross R, Giay T, Sastroamidjojo S, Schultink W, Lang NT. Premature complementary feeding is associated with poorer growth of Vietnamese children. J Nutr. 2000; 130(11):2683-90.

6. Victora CG, Vaughan JP, Lombardi C, Fuchs SMC, Gigante LP, Smith PG, et al. Evidence for protection by breastfeeding against infant deaths from infections by diseases in Brazil. Lancet. 1987; 2(8554):319-22.

7. World Health Organization. Collaborative Study Team on the Role of Breastfeeding on the prevention of Infant Mortality. Effect of breastfeeding on infant and child mortality due to infectious diseases in less developed countries: a pooled analysis. Lancet. 2000; 355(9202):451-5.

8. Zheng T, Holford TR, Mayne ST, Owns PH, Zhang $\mathrm{B}$, Boyle $\mathrm{P}$, et al. Lactation and breast cancer risk: a case control study in Connecticut. Br J Cancer. 2001; 84(11):1472-6.

9. Rosenblatt KA, Thomas DB. Lactation and risk of epithelial ovarian cancer. Int J Epidemiol. 1993; 22(2):192-97.

10. Commings RG, Klineberg RJ. Breastfeeding and other reproductive factors and risk of hip fracture in elderly women. Int J Epidemiol. 1993; 22(4): 684-91.

11. Pisacane A, Impagliazzo N, Russo M, Valiani R, Mandarini A, Florio $C$, et al. Breast feeding and multiple sclerosis. Br Med J. 1994; 308(6941): 1411-2.

12. Kennedy KI, Visness CM. Contraceptive efficacy of lactational amenorrhoea. Lancet. 1992; 339(8787): 227-30.

13. Giugliani ERJ. Amamentação exclusiva e sua promoção. In: Carvalho MR, Tamez RN. Amamentação: bases científicas para a prática profissional. Rio de Janeiro: Guanabara Koogan; 2002. p.11-24. 
14. World Health Organization. The optimal duration of exclusive breastfeeding. 2001. [cited 200318 Nov]. Available from: URL: http://www.who.int

15. Venâncio SI, Escuder MML, Kitoko P, Rea MF, Monteiro CA. Frequência e determinantes do aleitamento materno em municípios do Estado de São Paulo. Rev Saúde Pública. 2002; 36(3):313-8.

16. Brasil. Ministério da Saúde. Prevalência (\%) e intervalo de confiança de aleitamento materno exclusivo, segundo regiões e capitais - Brasil. 1999 [acesso em 17 nov 2003]. Disponível em: URL: http://www.aleitamento.gov.br

17. Rea MF. Reflexões sobre amamentação no Brasil: de como passamos a 10 meses de duração. Cad Saúde Pública. 2003; 19(1):537-45.

18. Carvalhaes MABL, Parada CMGL, Manoel CM, Venâncio SY. Diagnóstico da situação do aleitamento materno em área urbana do sudeste do Brasil: utilização de metodologia simplificada. Rev Saúde Pública. 1998; 32(5):430-6.

19. Costa ACM, Carvalhaes MABL, Parada CMGL. A prevalência do aleitamento materno em Botucatu, no ano de 1999. In: $12^{\circ}$ Congresso de Iniciação Científica da UNESP; 17-20 out 2000; Brasil. São Paulo: São José do Rio Preto; 2000. p.150.

20. Instituto Brasileiro Geografia e Estatística. Sinopse preliminar do censo demográfico. 2000 [acesso em 1 dez 2003]. Disponível em: URL: http://www. ibge.gov.br

21. Brasil. Ministério da Saúde. Informações de saúde - Brasil. 2004 [acesso em 19 jan 2004]. Disponível em: URL: http://www.datasus.gov.br

22. World Health Organization. Division of Child Health and Development. Indicators for assessing breastfeeding practices. Geneva; 1991.

23. Ferreira MU, Cardoso MA, Santos AL, Ferreira CS, Szarfarc SC. Rapid epidemiologic assessment of breastfeeding practices: probit analysis of current status data. J Trop Pediatr. 1996; 42(1):50-3.

24. Vieira S. Bioestatística: tópicos avançados. São Paulo: Atlas; 2004.
25. Lamounier JA. Tendências do aleitamento materno no Brasil. Rev Med Minas Gerais. 1999; 9(2): 54-65.

26. Montrone VC, Arantes CIS. Prevalência do aleitamento materno na cidade de São Carlos, São Paulo. J Pediatr (RJ). 2000; 76(2):138-42.

27. Audi CAF, Corrêa AMS, Latorre MRDO. Alimentos complementares e fatores associados ao aleitamento materno e ao aleitamento materno exclusivo em lactentes até 12 meses de vida em Itapira, São Paulo, 1999. Rev Bras Saúde Materno Infant. 2003; 3(1):85-93.

28. Woolridge MW, Phil D, Baum JD. Recent advances in breastfeeding. Acta Pediatr. 1993; 35(1):1-12.

29. Horta BL, Olinto MTA, Victora CG, Barros FC, Guimarães PRV. Amamentação e padrões alimentares em crianças de duas coortes de base populacional no Sul do Brasil: tendências e diferenciais. Cad Saúde Pública. 1996; 129(1):43-8.

30. Assis AMO, Prado MSP, Freitas MCS, Silva RCR, Ramos LB, Machado AD. Prática do aleitamento materno em comunidades rurais do semi-árido baiano. Rev Saúde Pública. 1994; 28(5):380-4.

31. Kitoko PM, Réa MF, Venâncio SI, Vasconcelos ACCP, Santos EKAS, Monteiro CA. Situação do aleitamento materno em duas capitais brasileiras: uma análise comparada. Cad Saúde Pública. 2000; 16(4):1111-9.

32. Caldeira AP, Goulart EMA. A situação do aleitamento materno em Montes Claros, Minas Gerais: estudo de uma amostra representativa. J Pediatr (Rio J). 2000; 76(1):65-72.

33. Passos MC, Lamounier JA, Silva CAM, Freitas SN, Baudson MFR. Práticas de amamentação no município de Ouro Preto, MG, Brasil. Rev Saúde Pública. 2000; 34(6):617-22.

Recebido em: 31/3/2005

Versão final reapresentada em: 21/8/2006 Aprovado em: 21/12/2006 
